Titre court : Usagers et déprescription médicamenteuse en EMS

\title{
Points de vue d'usagers sur la déprescription de médicaments en maison de retraite
}

\author{
Lucie LECHEVALIER HURARD
}

Sociologue, PhD, Assistante de recherche, Haute École de Santé Vaud (HESAV), Haute

École Spécialisée de Suisse Occidentale (HES-SO)

Damien CATEAU

Pharmacien, Institut des Sciences Pharmaceutiques de Suisse Occidentale (ISPSO), Université de Genève, Université de Lausanne

\section{Olivier BUGNON}

Co-chef du département des policliniques et pharmacien chef, Professeur associé, Centre universitaire de médecine générale et de santé publique (Unisanté), Université de

Lausanne ; Institut des Sciences Pharmaceutiques de Suisse Occidentale (ISPSO), Université de Genève, Université de Lausanne, Suisse

\section{Anne NIQUILLE CHARRIÈRE}

Pharmacienne cheffe adjointe, $\mathrm{PhD}$, Centre universitaire de médecine générale et de santé publique (Unisanté), Université de Lausanne, Suisse

\section{Rose-Anna FOLEY}

Anthropologue, PhD, Professeure HES associée, Haute École de Santé Vaud (HESAV), Haute École Spécialisée de Suisse Occidentale (HES-SO) ; Plateforme de Recherche Qualitative, Unisanté, Centre universitaire de médecine générale et de santé publique, Université de Lausanne, Suisse.

Résumé - Cet article est issu d'une recherche interdisciplinaire en pharmacie et sciences sociales sur la polymédication et la déprescription en maison de retraite menée en Suisse romande. Si les facteurs psychosociaux sont souvent mobilisés pour expliquer la difficile déprescription (réduction encadrée de la consommation de médicaments pour réduire ses effets délétères identifiés), les usagers interviewés mettent en lumière les facteurs structurels du fonctionnement des établissements dans la persistance de ce phénomène. Perdant leur autonomie sur beaucoup d'aspects de leur vie quotidienne, la plupart des résidents délèguent intégralement la gestion des médicaments aux professionnels et estiment ne plus vraiment avoir de prise sur l'évolution des prescriptions. L'enquête montre pourtant qu'entre recherche d'efficacité et crainte des effets secondaires, les usagers se révèlent perméables aux discours des professionnels et accessibles à la sensibilisation aux risques de la polymédication. L'article conclut sur des pistes qui pourraient faciliter une démarche de déprescription concertée, et favoriser le partage des décisions avec les résidents de maisons de retraite à partir du levier des consommations de médicaments.

Mots clés - maison de retraite, médicaments, personnes âgées, polymédication, déprescription 


\section{Abstract - Residents and families' points of view on deprescribing in NH's}

Based on an interdisciplinary research in pharmacy and social sciences on deprescribing in Western Switzerland, this manuscript analyzes the points of view of nursing homes' residents and their relatives regarding their medication use.

If psycho-social factors are often considered to explain the impediments of medication reduction despite identified noxious effects, the interviewed NH's residents highlight structural factors in the nursing homes, which are held responsible for the phenomenon's persistence.

Loosing their autonomy in many areas of their daily lives when entering the nursing home, most residents entirely delegate the medication management to health professionals and consider they do not have control on the prescriptions' evolution.

This study shows that between their quest for efficiency and fears of side effects, NH's residents are permeable to health professionals' discourses and open to risk sensitization regarding polymedication. This article concludes on tangible directions enabling a concerted deprescribing plan favoring shared decisions with nursing home residents based on the leverage of medication use.

Keywords - Nursing homes, medicines, elderly, polymedication, deprescribing

\section{$\underline{\text { Introduction }}$}

\section{Une recherche interventionnelle sur la déprescription}

La réflexion proposée dans cet article provient d'une collaboration interdisciplinaire entre des chercheurs en pharmacie communautaire et des chercheuses en sciences sociales au sein d'un programme de recherche interventionnelle concernant la consommation de médicaments des personnes âgées en maison de retraite. Les sciences sociales ont été mobilisées dans le cadre de cette recherche interventionnelle pour évaluer dans le contexte d'Établissements Médico-Sociaux (EMS) hébergeant des personnes âgées de deux cantons suisses romands (Vaud et Fribourg) la faisabilité et l'acceptabilité de la déprescription.

Une enquête qualitative a été réalisée auprès de 3 groupes professionnels impliqués en maison de retraite (médecins, pharmaciens, infirmiers) ainsi que des résidents et leurs proches, pour identifier, dans la lignée d'autres travaux sur les professionnels et sur les résidents (Anderson et al., 2014 ; Luymes et al., 2016) leurs perceptions et leurs ressentis vis-à-vis de la déprescription, et chercher les leviers sur lesquels agir pour accompagner la déprescription en vue d'une réduction du risque de iatrogénie liée à la polymédication en maison de retraite.

\section{État de la recherche}

La déprescription est définie comme «le processus, supervisé par un professionnel de soin, de retrait de médicaments dont les risques potentiels sont supérieurs aux bénéfices attendus, en vue d'une réduction de la polymédication et de ses conséquences » (Reeve, 2015, p.1266). II s'agit d'une démarche de réduction de la polymédication dont les risques sont considérés comme importants par les professionnels de santé. Ces risques sont d'abord liés à l'utilisation de médicaments potentiellement inappropriés définis dans des listes ${ }^{1}$ nationales, régulièrement mises à jour en fonction de l'évolution des données sur leurs effets. Ces risques sont ensuite liés aux interactions entre les médicaments, qui croissent avec le nombre de molécules consommées de manière concomitante. L'incertitude est partagée par de nombreux professionnels rencontrés, qui considèrent qu'à partir d'un certain nombre de médicaments on perd la maîtrise des effets. Or, avec l'augmentation de l'espérance de vie et l'évolution du rapport au vieillissement, les personnes âgées sont progressivement devenues une population médicale à part entière, pour qui des traitements sont de plus en plus mobilisés. Alors qu'elles avaient pu être l'objet d'abandon thérapeutique lorsqu'elles présentaient certaines maladies comme le cancer (Fentiman et al., 1990), les personnes âgées sont désormais plus souvent traitées. En témoigne le développement de spécialités médicales dédiées aux personnes âgées, telle que l'oncogériatrie (Extermann, 2010) et le fait que les personnes âgées reçoivent plus de médicaments qu'auparavant, destinés à traiter une grande variété de symptômes.

La polymédication peut être source de dégradation de l'état de santé, d'altération de la qualité de vie et d'augmentation des risques d'hospitalisation et de décès (Lau et al., 2005 ; Perri et al., 2005). Par ailleurs, ces problèmes de santé représentent des coûts supplémentaires (Joël, 2002 ; Lachamp et al., 2012). D'abord, les systèmes d'assurance sociale assument le coût direct des nombreux médicaments consommés. Mais il faut y

\footnotetext{
${ }^{1}$ La liste de Mark H. Beers (1991) et STOPP/START par exemple.
} 
ajouter aussi les coûts des éventuels accidents de santé, qui débouchent notamment sur des hospitalisations et des prises en charge médico-sociales, ainsi que l'augmentation de la dépendance résultant de ces accidents.

D'un point de vue pharmacologique, réduire le nombre de molécules en « faisant le ménage » et en retirant des prescriptions les médicaments sujets à caution permet de limiter le risque d'éventuelles interactions néfastes.

\section{Méthodologie de l'enquête}

Une partie de l'enquête, présentée ici, s'est intéressée au point de vue des résidents de maisons de retraite et de leurs proches. Entre 2017 et 2018, 12 entretiens ont été réalisés dans 4 maisons de retraite différentes : 11 l'ont été avec des résidents (dont 3 étaient accompagnés d'un ou deux proches), et un a été réalisé séparément avec la fille d'une résidente. Les cantons de Vaud et Fribourg sont représentés équitablement dans l'échantillon, à hauteur de 6 entretiens de chaque côté.

Les questions posées aux participants ont porté sur leur parcours de santé et notamment les raisons qui les ont conduits en maison de retraite, leurs pratiques et représentations autour de leur consommation de médicaments souvent en lien avec l'accompagnement professionnel, et de l'entourage dont ils bénéficient.

Les critères d'inclusion étaient d'être âgé de plus de 65 ans, de résider en établissement depuis au moins 4 mois (situations stabilisées) et d'avoir au moins 5 traitements réguliers prescrits par un médecin.

Une autre partie de l'enquête a porté sur le point de vue des différents professionnels intervenant dans la prescription et la gestion des médicaments en maisons de retraite (médecins, infirmiers, pharmaciens) (Foley et al, 2020).

La participation à l'enquête n'a fait l'objet d'aucune rétribution.

Tous les noms des participants dont les propos apparaissent dans le texte ont été anonymisés.

L'étude a été financée par le Fonds National de la Recherche Suisse au travers du Programme National de Recherche 74 "Smarter Health Care"2. La Commission Cantonale d'Éthique de la recherche sur l'être humain du Canton de Vaud (CER-VD) en a approuvé le protocole de recherche en avril 2017 (numéro d'approbation 201700211).

\section{Des facteurs structurels déterminants pour les résidents et de leurs proches}

Les résidents et leurs proches mettent en évidence les facteurs structurels qui influencent selon eux fortement leur rapport aux médicaments, tout particulièrement dans le contexte de la maison de retraite, où la gestion des médicaments est quasiment intégralement assurée par les professionnels. Nous appuyons nos analyses sur les travaux d'anthropologie du médicament le considérant comme un objet pharmacologique simultanément investi de sens et comme un important médiateur du rapport à soi, aux autres et au monde (Fainzang, 2001 ; Van der Geest et Whyte, 1989). Dans une première partie, nous explorons le sentiment exprimé par les usagers d'une dépossession de leur rapport aux médicaments à l'entrée en maison de retraite.

Dans une seconde partie, notre attention se porte plus spécifiquement sur le point de vue des résidents vis-à-vis de l'arrêt éventuel de certains de leurs médicaments.

\section{La maison de retraite : une institution structurante dans la consommation de médicaments}

Les usagers rencontrés dans le cadre de cette recherche mettent en avant le fait que leurs consommations de médicaments sont loin d'être librement choisies, et qu'au contraire, elles sont fortement déterminées par la maison de retraite. Comme l'a montré l'étude de Delphine Dupré-Leveque (2002) sur la consommation des médicaments des personnes âgées en maison de retraite en France, les personnes âgées sont déchargées de leurs médicaments dès leur entrée en institution. Parce qu'elle constitue autant un lieu de soin qu'un lieu de vie, la maison de retraite apparaît alors comme un exemple de ce qu'Erving Goffman (2005) appelle « une institution totale » en ce qu'elle gère la totalité des besoins et des aspects de la vie quotidienne des personnes dont elle s'occupe. La gestion des traitements y est centralisée. Les résidents interviewés font part justement du changement important qu'a constitué l'entrée en maison de retraite dans leurs rapports aux médicaments et à leur gestion.

\section{"Quand il y a un problème, j'en fais part aux infirmières » : un suivi médical par procuration}

Le premier changement important concerne la relation avec le prescripteur. Contrairement au suivi à l'extérieur qui se fait sous la forme de consultations, les rencontres avec le médecin ne sont plus systématiquement nécessaires 
pour envisager des modifications des traitements. La décision n'implique plus forcément la consultation en face-àface entre le patient et son médecin, et les infirmiers jouent un rôle de médiateur dans les deux sens. Ils reçoivent la requête, et la font remonter au médecin. Le résident est ensuite informé par les infirmiers de la décision prise par le médecin. Il arrive aussi que le résident constate simplement la modification du traitement à la distribution suivante, sans en avoir été spécifiquement informé. Les résidents et leur entourage sont habitués à cette procédure :

Si [ma mère] se plaint qu'elle a mal vraiment quelque part, moi je fais part aux infirmières. (Fille de Mme K.)

\section{«Ils ont pris les choses en main à leur manière » : une situation d'observance forcée}

Le deuxième changement concerne la capacité d'adaptation du traitement par le résident. Quand elles vivent à domicile, les personnes conservent une certaine liberté de prendre ou pas les médicaments qui leur sont prescrits, ou de jouer sur les doses et les moments de prise. C'est ce que Carmel Hughes et Roz Goldie (2009, p. 506) appellent la « non-observance intelligente » ou « non observance volontaire », ou que Sjaak Van der Geest et Susan Reynolds Whyte (1989), dans leur article fondateur de l'anthropologie du médicament, considéraient comme le caractère démocratique du médicament qui permet de s'affranchir de la relation et de la dépendance au thérapeute. En maison de retraite, les personnes âgées se trouvent dans une situation qu'on pourrait qualifier à l'inverse d' " observance forcée " vis-à-vis des médicaments qui leur sont prescrits. Les infirmiers assurent la distribution des traitements à horaires réguliers et en vérifient la bonne prise. Dans ces circonstances, il n'y a pas ou peu d'oubli et d'adaptation possible.

Pour Mme C., l'entrée en maison de retraite a représenté une surmédicalisation des petits problèmes de santé bénins. Elle la regrette, sans parvenir à la contester.

Je prenais le minimum de médicaments et je me soignais homéopathiquement. Puis ici quand je suis arrivée, on m'a tout balancé. [...] Ils ont pris les choses en main à leur manière. [...] [Avant je prenais] ces trucs contre les grippes, les rhumes. [...] Dès qu'il y avait quelque chose, j'avais un petit coffret avec plusieurs trucs. [...] J'ai essayé [de négocier mais] on n'a pas le droit d'avoir des médicaments.

La fille de Mme L. témoigne aussi des changements de médication intervenus pour sa mère.

[Ma mère] a l'habitude du sirop pour la toux, ça la calme aussi psychologiquement, elle a l'impression que ça va mieux, donc pourquoi pas? [...] J'ai vu que [les infirmiers] aimaient pas trop [...]. Peut-être qu'ils pensent que ça sert à rien! Elle a eu plusieurs fois des antibiotiques [depuis qu'elle est dans l'établissement] [...]. Mais avant, elle prenait pas d'antibiotiques.

La littérature médicale montre que les consommations de médicaments diffèrent en fonction du lieu de vie des personnes et qu'il existe des traditions de prescription différentes entre le domicile et les maisons de retraite (Johnell et Fastbom, 2012). Du point de vue des résidents interviewés, l'entrée en maison de retraite peut donc représenter des changements importants dans la médication, sans qu'ils aient été forcément négociés et acceptés par eux.

\section{« J'avale ce qu'on me donne » : un fort sentiment de dépossession de soi}

Les changements de traitements, en particulier lorsqu'ils ne sont pas négociés progressivement, contribuent pour une partie des résidents interviewés à un sentiment de dépossession de soi, qui s'exprime à propos de la consommation de médicaments mais aussi plus largement de la vie dans l'établissement. Comme l'observe Delphine Dupré-Leveque (2002), les personnes âgées sont dépossédées de leur rapport intime aux médicaments, souvent maintenu intact jusque-là au domicile. Néanmoins, en parlant de leurs traitements médicamenteux, les résidents interrogés abordent en réalité beaucoup plus globalement leur rapport à la maison de retraite et à la vie qu'ils y mènent, à propos de laquelle certains expriment un sentiment très fort de « dépossession de soi ». Cette notion désigne "l'expérience subjective des personnes privées de leurs instruments de décision, y compris les plus intimes » (Weber, 2012, p. 329) dans un contexte de délégation des actes de la vie quotidienne, liée à la perte d'autonomie. Elle décrit bien l'expérience de la vie en maison de retraite où l'activité des professionnels est structurellement tournée vers la gestion des besoins corporels (Rimbert, 2010, p. 187), malgré la revendication de l'importance de faire des établissements gériatriques des « lieux de vie » respectant la subjectivité des personnes qui vont y passer les dernières années de leur vie.

Mme K., qui souffre de démence, exprime nettement un sentiment de perte complète d'autonomie depuis qu'elle est entrée en maison de retraite, où sa vie est régentée par le rythme et les obligations de l'institution.

Moi je voudrais pouvoir faire ma toilette et puis après sortir quand j'en ai envie ! [...] Pas qu'on me dise " aujourd'hui vous irez telle et telle place " [...] Surtout, [...] il y a toujours une infirmière ou bien une fille qui vient avec moi... [...] Je peux pas aller toute seule, c'est ça qui me gêne. [...] "vous devez... obéir " voilà ce qu'on me dit. Alors bon, je me tais [...] je dois obéir à passé 80 ans ? [...] Quand on est gamins on doit obéir à nos parents, après on doit obéir aux patrons. Après on va dans un Home comme ici, ben... on doit obéir en somme !

Mme $\mathrm{K}$ lie ce sentiment à une défiance et une contestation de la pertinence des médicaments qui lui sont donnés. 
L'infirmière elle vient et puis elle me dit « Mme K. aujourd'hui faut prendre ce médicament » [...] Mais pourquoi je prends un médicament? "C'est parce que vous avez du mal à penser normalement ». [...] Mais je suis quandmême pas toc ou quoi ? Je suis pas malade autrement. " Non mais c'est pour votre bien ». C'est souvent ce qu'on me dit.

D'ailleurs, le dispositif d'enquête, qui a comparé les médicaments connus et signifiants pour les personnes interviewées avec la liste des médicaments effectivement distribués chaque jour, a permis de mettre en évidence la méconnaissance par une partie importante des résidents des médicaments qu'ils consomment quotidiennement. En particulier, un tiers des personnes de l'échantillon indiquent n'avoir aucune idée ou seulement une idée très imprécise des traitements qui leur sont distribués chaque jour.

Tous ces petits médicaments que je prends, je sais pas pourquoi c'est. [...] pourquoi pas dire [à quoi servent ces médocs] et puis on en parle plus. Après on sait ce que c'est. Mais pas cacher comme ça des choses. [...] J'ai pas eu de réponse on m'a rien dit, rien." (Mme D.)

J'avale ce qu'on me donne! [...] sans réfléchir des fois [...] Des fois est-ce que je devrais avoir moins ou un de plus, je sais pas ! Parce qu'ils t'expliquent pas. Ils te disent pas ce que c'est, pourquoi... (Mme J.)

II est à noter que les sentiments de méconnaissance et de suspicion vis-à-vis des médicaments consommés sont concentrés parmi les personnes de l'échantillon pour lesquelles l'entrée en maison de retraite n'a pas été véritablement choisie, ou pour lesquelles elle a été mal vécue.

Ceux qui ont fait le choix volontaire d'entrer en établissement expriment plus régulièrement leur sentiment d'avoir la capacité de négocier leurs traitements, pour rendre leur quotidien plus confortable et autonome par rapport au fonctionnement rigide de l'institution. Mme G. négocie notamment certains horaires de prise de ses médicaments et se voit autorisée à gérer seule leur administration lorsqu'elle sort de l'établissement pour la journée :

Je pars assez souvent [...] un jour entier. [...] Ils peuvent me donner tout le paquet un jour, je sais quand je dois les prendre.

II me demande le docteur, il me pose la question et moi je lui dis « c'est mieux de prendre à telle heure ». II m'écoute.

On retrouve ici nettement la congruence mise en évidence par Johanne Collin à propos du domicile entre confiance ou défiance vis-à-vis du médicament et vis-à-vis du système de santé (Collin, 2002, p. 153), et ce de manière d'autant plus marquée que l'établissement est à la fois le décideur, le pourvoyeur et le distributeur des médicaments.

\section{«On en avait juste parlé en passant » : des proches éloignés de la décision sur les médicaments}

Les professionnels interrogés dans l'autre volet de la recherche considéraient que les proches de résidents tendaient à être réticents à retirer des prescriptions (Foley et al, 2020). Ils imputaient la difficulté à déprescrire, audelà de la complexité des situations cliniques due à l'allongement de l'espérance de vie, et de l'augmentation des polymorbidités, principalement à l'attachement fort des résidents et de leurs proches à leurs médicaments, à leur incapacité de changement ou encore à des craintes d'abandon thérapeutique ou de désinvestissement des professionnels vis-à-vis d'eux. L'enquête sur les usagers révèle que cette hypothèse est en grande partie erronée. Les proches se révèlent particulièrement éloignés de la décision médicale concernant les médicaments. Au moment du recrutement pour l'enquête, trouver des proches pour les entretiens a été difficile. Aux dires de l'infirmier-chef d'un des établissements contactés, aucune famille ne pouvait être considérée comme participant aux décisions de soin, prises de fait par le médecin de l'établissement en lien avec l'équipe soignante. Le fils d'une résidente a d'ailleurs décliné la proposition, puisqu'il n'avait aucune idée des médicaments consommés par sa mère et bien qu'il soit par ailleurs très présent dans les relations avec l'établissement.

Cette absence d'implication dans les décisions de traitements s'est confirmée dans les quatre entretiens qui ont impliqué des proches. «On n'est jamais là » : depuis que sa mère est entrée en maison de retraite, la fille de Mme J. ne connaît plus ses médicaments, d'autant plus qu'elle n'a jamais l'occasion d'assister au moment de la prise. Elle n'a un regard là-dessus que lorsqu'elle emmène sa mère déjeuner à l'extérieur et qu'elle lui administre au repas les médicaments remis par l'équipe soignante.

C'est seulement dans certains tournants importants de la médication qu'elle est prévenue a posteriori de ce qui a été mis en place.

Une fois ils avaient rajouté de la morphine. Ça avait des effets secondaires de nausées [...] ça, elles m'en avaient parlé les infirmières [...] c'est le médecin qui prescrit. Mais elles sont en contact direct avec lui et puis, nous on en avait juste parlé en passant. (Fille de Mme J.)

Notons que la fille de Mme J. ne se plaint pas de cette relative mise à l'écart, et ne revendique pas d'être consultée pour ce type de décisions.

II en va différemment de la fille de Mme L. Elle est l'interlocutrice des professionnels qui entourent sa mère dans l'établissement, où elle vient pour des visites presque tous les jours. Elle est au fait des évolutions récentes de 
traitement. Pour répondre à la demande de l'enquêtrice, elle demande à l'infirmière la liste des prescriptions en cours, et découvre à cette occasion deux médicaments supplémentaires dont elle n'avait pas connaissance : un laxatif et un hypnotique. Elle fait part de son grand mécontentement à l'infirmière, et réclame que sa demande d'être tenue au courant de tout changement de traitement soit strictement respectée.

Cette situation montre que les proches même les plus investis ne sont pas forcément informés des traitements à vocation institutionnelle, c'est à dire destiné à préserver l'ordre de l'établissement (Lechevalier Hurard, 2013). En l'occurrence, l'hypnotique contribue probablement à aider à l'endormissement d'une résidente désorientée et agitée, qui déambule la nuit, quand le personnel est moins nombreux et peut plus difficilement s'en occuper. II ne s'agit donc pas d'un traitement destiné à régler un problème de santé individuel. Plusieurs des membres de l'entourage rencontrés expriment d'ailleurs une préoccupation générale face à d'éventuelles utilisations abusives par l'établissement des médicaments qui « shootent », sans pour autant la constater chez leur parent.

Cette première partie de l'analyse a permis de mettre en évidence le fait que dans le contexte de la maison de retraite, les usagers, résidents et entourage, se sentent finalement peu mobilisés dans la gestion et les décisions autour des médicaments. Si certains se satisfont de cette situation, d'autres se plaignent d'une absence d'autonomie qui n'est que la métonymie d'une dépossession plus vaste valant pour l'ensemble des aspects de leur vie quotidienne, désormais déléguée à la maison de retraite.

Si elle était jugée nécessaire, la mise en place d'une démarche de déprescription pourrait donc se faire à la seule initiative des professionnels. Dans la mesure où ils disposent d'une situation privilégiée pour observer l'état de santé des résidents et ses évolutions, prescrire, distribuer et s'assurer de la prise effective des médicaments, ils peuvent a priori sans difficulté faire évoluer les prescriptions de façon à limiter les risques induits par la polymédication. Mais le processus de déprescription, s'il s'appuie sur la perception qu'ont les usagers de leurs consommations de médicaments, peut aussi contribuer à renforcer l'accompagnement centré sur le patient (patient centered care). Contrairement au regard professionnel sur cette question, les usagers rencontrés expriment de véritables questionnements quant à l'utilité, l'efficacité et la nocivité des traitements qu'ils consomment quotidiennement. Une démarche de déprescription concertée, prenant appui sur ces questionnements, nous semble constituer à la fois une piste à explorer pour réduire les risques de la polymédication et un excellent levier pour impliquer les résidents dans les décisions de santé qui les concernent, et aller à l'encontre du sentiment si prégnant de dépossession de soi.

\section{Vers une démarche de sensibilisation aux risques de la polymédication}

\section{« Je suis pas une boîte à pilules » : prise de distance avec l'image d'un rapport abusif aux médicaments}

Les points de vue des personnes interviewées contredisent souvent la représentation populaire selon laquelle les personnes âgées auraient un rapport abusif ou inadéquat aux médicaments (Collin, 2002, p. 151). Elles jugent la quantité de médicaments qu'elles consomment globalement importante.

J'en prends tellement! (Mme F.)

Je trouve que j'en ai trop de médicaments. (Mme D.)

Elles évoquent pourtant une certaine aversion à l'idée d'en consommer. "Je ne suis pas très médicament », dit Mme A. qui ne se considère pas comme " une boite à pilule », ni comme " une pharmacie ambulante ».

On n'est pas portés sur le médicament absolument à chaque fois.... Si nécessaire oui. (Mari de Mme F.)

Je souhaite qu'on ne consomme aucun médicament si c'est possible! Maintenant si l'effet du médicament est bénéfique, alors évidemment on est content qu'il soit là ! Mais sur le principe, on n'a pas de raison de consommer un médicament dont on n'a pas besoin. (Fille de Mme L.)

Évoquer le poids que représente une consommation importante de médicaments dans leur quotidien contribue à cette prise de distance.

Il y a eu une période où c'était vraiment très très très difficile. Elle n’arrivait pas à les avaler; Mme $F$. : La catastrophe. [les pilules] ne descendaient pas. [...] c'était physiologique, ça n'arrivait pas à descendre. (M. F.)

« J'en ai marre », dit Mme C. de la visite d'un infirmier 10 fois par jour dans sa chambre pour l'application de gouttes dans les yeux.

Plusieurs participants font aussi valoir des manières alternatives de prendre soin de leur santé, par exemple à travers l'exercice physique ou la surveillance de leur alimentation. Mme D. ne mange « rien que la moitié du repas » 
et ne prend pas de dessert pour contrôler son diabète et son cholestérol. Mme C. voit l'efficacité de l'exercice physique sur ses douleurs quand les médicaments ne sont pas forcément aussi profitables:

Je me demande si le Liorésal est nécessaire parce que c'est pour éviter de souffrir, mais je souffre quand même. [...] Là, ça va parce que j'ai fait des trucs, ce matin. J'ai eu ce monsieur de la locomotion, il m'a fait faire deux fois le tour de l'enceinte de l'EMS. Souvent je fais des navettes [avec le déambulateur], là, sur cette terrasse.

Entre condamnation du « trop de médicaments » et justification des molécules « vitales » ou « obligatoires » dans leur état, parce qu'ils soulagent et rendent leur quotidien vivable, les usagers évoquent bien ici le rapport ambivalent aux médicaments que décrit Johanne Collin chez des personnes âgées vivant à leur domicile (2002, p. 148).

\section{«Tant que ça va bien, je vais pas arrêter » : l'importance du sentiment d'efficacité}

Les personnes rencontrées jugent de la pertinence d'un médicament dans leur plan de traitement sur la base de deux critères : leur ressenti de son efficacité et leur tolérance à ses effets secondaires.

Lorsque l'entretien aborde la question de l'arrêt éventuel d'un médicament, ce qui revient avant tout est le sentiment d'efficacité immédiate des médicaments sur un symptôme : ceux qui améliorent de manière évidente un problème de santé sont plébiscités.

Parmi les médicaments qui paraissent indispensables à Mme E., on trouve un antidépresseur dont elle a expérimenté l'arrêt précédemment, lors d'une hospitalisation.

À [l'hôpital] ils m'ont enlevé mes antidépresseurs et puis ici il a fallu les remettre parce que j'étais bien en allant làbas et je suis ressortie complètement malade.

La crainte d'une rechute de sa dépression est très forte et l'incite à refuser toute modification de son traitement. Cette peur de la réapparition du symptôme fait partie des arguments principaux que la littérature médicale identifie d'ailleurs comme barrière à la déprescription (Reeve et al., 2013, p. 799), et en particulier dans le cas des médicaments psychotropes (Verbeek-Heida et Mathot, 2006).

Mais le sentiment d'efficacité tient parfois aussi à la seule absence de dégradation.

Les gouttes dans les yeux, je pense que ça, c'est indispensable. Ça va pas me guérir mais disons que ça empêche peut-être au glaucome de progresser je pense. [...] Ça le stabilise parce que je vois pas de différence. Depuis qu'on a commencé à mettre les gouttes, je peux pas dire 'je vois mieux' ou 'je vois plus du tout' ou 'moins bien'. (Mme A.)

M. I. a, lui, intégré la dimension préventive de certains de ses traitements : se « défendre contre les autres [médicaments] » est très important pour lui, en particulier car ses traitements anti-Parkinson font, d'après lui, courir des risques à son estomac.

Pour le moment ça va bien, je touche du bois! Tant que ça va bien, je vais pas arrêter [le médicament pour l'estomac].

Comme le rappelle Johanne Collin, « le terme médicament, du terme pharmakon, à la fois remède et poison est souvent appréhendé en fonction du versant négatif de sa double identité et associé à des risques d'empoisonnement » (2002, p. 147).

Plusieurs des résidents interviewés se satisfont des médicaments qu'ils prennent dans la mesure où ils disent les " supporter », c'est à dire parvenir à encaisser leur double caractère, à la fois thérapeutique et néfaste. Pour eux, l'absence d'effets secondaires constitue la preuve que le médicament est adapté, en comparaison avec d'autres, qu'ils ont reçus précédemment et qui ont donné lieu à des expériences désagréables.

Je supportais pas [la morphine]. Et d'autres médicaments qui sont très forts, je supportais pas. Du coup on savait plus quoi faire. (Mme E.)

L'apparition d'effets secondaires constitue d'ailleurs une cause déterminante pour envisager l'arrêt d'un médicament pour les personnes interviewées. Mme G. par exemple serait prête à essayer d'arrêter un médicament contre la douleur « pour être moins somnolente ».

Dans d'autres cas, les proches se demandent si c'est le médicament ou les symptômes de la maladie qui dégradent le résident:

Peut-être c'est les médicaments qui t'assomment ! (Fille de Mme J.)

Pour nous en tout cas c'est difficile de juger si le manque de mémoire, c'est à cause des médicaments [...] ou bien c'est la mémoire qui déjà simplement n'est pas bonne. Et puis il y a l'âge. (Gendre de Mme J.)

\section{"C'est lui qui dirige ce qu'il faut prendre ": Une forte remise de soi vis-à-vis du prescripteur}


Malgré ces doutes, dans l'ensemble les résidents et leurs proches s'en remettent aux choix du prescripteur, estimant qu'ils ne disposent pas du savoir nécessaire pour juger de l'effet des médicaments. Après avoir émis son avis lors de l'entretien sur un éventuel arrêt d'un médicament qui la rend somnolente, Mme G. se retranche derrière son sentiment de méconnaissance :

Maintenant, je ne suis pas pharmacienne!

Généralement, ils se plient aux choix de traitements qui sont préconisés par leur prescripteur habituel, sans les questionner sur le fond, surtout s'il s'agit d'un spécialiste qui les suit depuis longtemps.

On se pose des questions, on se dit "Est-ce que ça sert encore ? " [...] Quand j'allais chez le Docteur Y. on en discutait. Des fois qu'il pourrait enlever un, enlever deux et puis... une fois il m'avait dit "Non, ça il faut le garder » ou « Ça vous l'enlevez ». (M. I.)

Pour M. I., le choix final revient toujours à son médecin.

\section{Une méconnaissance des éventuelles interactions médicamenteuses}

Ces entretiens témoignent donc d'une confiance de type «clérical » (Hammer, 2004, p. 95) des résidents vis-à-vis des prescriptions qui leur sont faites, qui se caractérise par une certaine docilité et une délégation de la responsabilité au médecin dans le cadre d'une division de tâches stricte et d'une asymétrie des savoirs. Ils perçoivent la nocivité potentielle des médicaments: cependant ils associent principalement les symptômes désagréables à la maladie et la préservation ou l'amélioration de leur état de santé au médicament.

Aucun des résidents ou des membres de leur entourage n'évoque en revanche, pour des médicaments bien tolérés au moment de l'entretien, une crainte quant à ses effets sur le long terme. La notion d'interactions néfastes entre plusieurs médicaments est, elle aussi, complètement absente de leur propos alors que ces craintes de sécurité sont très présentes chez les soignants. Si le prescripteur a jugé le médicament nécessaire, il n'y a pas de raison pour eux de le remettre en cause. Dès lors qu'ils ressentent physiquement l'efficacité du médicament et que celui-ci ne provoque pas d'effets secondaires insupportables, les résidents le considèrent comme adapté et acceptent d'assumer le poids de sa prise, même si cela pèse sur leur quotidien et/ou sur leurs finances.

Les usagers jugent donc plutôt l'intérêt et les inconvénients de leurs médicaments séparément les uns des autres. Le fait que la polymédication représente un risque potentiel du fait des synergies incertaines entre les molécules, apparait trop abstrait pour entrer en compte dans leurs critères de jugement.

\section{Conclusion}

Pour avancer sur la réduction de la polymédication et éviter de se limiter aux facteurs psycho-sociaux des résidents dans l'acceptation de la déprescription il semble qu'il appartient aux professionnels de santé de travailler à la prise de conscience par les usagers de la dimension du risque potentiel des médicaments qu'ils consomment. Un travail pédagogique sur la nocivité de la polyconsommation pourrait être efficace, à condition de prendre en compte l'historique et le rapport individualisé des personnes à leurs médicaments.

L'enquête menée montre que les résidents sont extrêmement perméables au discours des prescripteurs (en particulier sur la nécessité du soin et de la prévention) et qu'ils seraient vraisemblablement réceptifs à des arguments sur la nocivité, d'autant plus qu'ils ont, pour la plupart, déjà expérimenté des effets secondaires négatifs de certains traitements. Ces expériences vécues de symptômes indésirables, le ressenti physique de la nocivité, peuvent constituer des supports solides pour initier une démarche pédagogique. Pour ne pas devenir anxiogène, cette dernière se doit de ne pas se centrer uniquement sur les risques, mais aussi de proposer des alternatives, non médicamenteuses, aux problèmes de santé rencontrés et au besoin de prévention.

D'autres paramètres peuvent faciliter la mise en place de la déprescription. Comme l'ont montré d'autres études, la réversibilité du processus est essentielle pour qu'il soit accepté : le traitement doit pouvoir être repris en cas de retour des symptômes (Reeve et al., 2013, p. 81). Dans notre enquête, la fille de Mme L. le confirme :

Je dirais d'accord [pour l'arrêt d'un médicament], on surveille on regarde ce qui se passe et puis le cas échéant... ça va bien ou sinon on le redonne!

Le travail avec l'entourage semble indispensable, en particulier concernant les résidents présentant des troubles cognitifs, puisque l'enquête révèle qu'ils sont trop souvent laissés complètement à l'écart des décisions de santé, qu'ils ne découvrent qu'après coup.

Ce travail de pédagogie pourrait être éventuellement pris en charge en collaboration avec les pharmaciens. En tant que spécialistes du médicament et extérieurs à l'institution, ils seraient susceptibles d'être un interlocuteur original, apportant une triangulation dans le rapport entre résidents et médecins, pas toujours très fluide. II y a là un travail important à faire pour permettre aux usagers d'avoir recours à ces interlocuteurs, et aux professionnels de s'appuyer sur eux. 
Mais si la démarche de sensibilisation des résidents est essentielle pour garantir que l'évolution de la consommation médicamenteuse se fasse en concertation étroite avec la personne la plus concernée, rappelons que la prescription reste une affaire de professionnels : ce sont en effet les médecins qui décident en dernier recours de ce qui figure sur l'ordonnance. L'autre volet de la recherche a d'ailleurs mis en évidence une série de points sur lesquels il est possible d'agir du côté des professionnels pour favoriser la déprescription : d'abord dégager et financer du temps pour le travail d'analyse des prescriptions, ensuite mobiliser des moyens pour assurer la stabilité et la cohérence des équipes de soin, dans la perspective de favoriser la fluidité des échanges d'informations et d'expertise (Foley et al, 2020). Notre recherche interventionnelle en cours œuvre dans le sens d'une mise en place concertée et sécurisée de mesures de déprescription médicamenteuses au sein de maisons de retraite de Suisse romande.

Gageons qu'une mobilisation conjuguée du côté des usagers comme de celui des professionnels contribuera certainement à une réduction de la iatrogénie liée à la polymédication en maison de retraite, mais aussi à la promotion de la bientraitance et de la dignité de la personne. 


\section{Références}

Anderson, K., Stowasser, D., Freeman, C. et Scott, I. (2014). Prescriber barriers and enablers to minimising potentially inappropriate medications in adults: A systematic review and thematic synthesis. BMJ Open, 4(12). doi: 10.1136/bmjopen-2014-006544

Beers, M. H., Ouslander, J. G., Rollingher, I., Reuben, D. B., Brooks, J. et Beck, J. C. (1991). Explicit criteria for determining inappropriate medication use in nursing home residents. UCLA Division of Geriatric Medicine. Archives of Internal Medicine, 151(9), 1825-1832.

Collin, J. (2002). Observance et fonctions symboliques du médicament. Gérontologie et société, 25(103), $141-159$. doi : $\underline{10.3917 / \text { gs. } 103.0141}$

Dupré-Lévêque, D. (2002). Le médicament : Un outil de communication ? Gérontologie et société, 25(103), $161-176$. doi: $10.3917 / g s .103 .0161$

Extermann, M. (2010). Geriatric oncology: An overview of progresses and challenges. Cancer Research and Treatment: Official Journal of Korean Cancer Association, 42(2), 61-68. doi: https://doi.org/10.4143/crt.2010.42.2.61

Fainzang, S. (2001). Médicaments et société : Le patient, le médecin et l'ordonnance. Paris, France : Presses universitaires de France.

Fentiman, I. S., Tirelli, U., Monfardini, S., Schneider, M., Festen, J., Cognetti, F. et Aapro, M. S. (1990). Cancer in the elderly: Why so badly treated? Lancet, 335(8696), 1020-1022. doi: 10.1016/0140-6736(90)91075-I

Foley R-A., Lechevalier Hurard L., Cateau D., Koutaissoff D., Bugnon O., Niquille A. (2020). Physicians', Nurses' and Pharmacists' Perceptions of Determinants to Deprescribing in Nursing Homes Considering Three Levels of Action: A Qualitative Study, Pharmacy 8(1), 17; https://doi.org/10.3390/pharmacy8010017

Goffman, E. (2005). Asiles : Études sur la condition sociale des malades mentaux et autres reclus. Paris, France : Éditions de Minuit.

Hammer, R. (2004). La confiance dans le médecin et les formes de son autorité. Carnets de bord, (7), 94-100.

Hughes, C. M. et Goldie, R. (2009). «I just take what I am given »: Adherence and resident involvement in decision making on medicines in nursing homes for older people: A qualitative survey. Drugs \& Aging, 26(6), 505-517. doi: $10.2165 / 00002512-200926060-00007$

Joël, M.-È. (2002). La consommation de médicaments des personnes âgées : Éléments d'analyse économique. Gérontologie et société, 25(103), 29-38. doi: 10.3917/gs.103.0029

Johnell, K. et Fastbom, J. (2012). Comparison of Prescription Drug Use between Community-Dwelling and Institutionalized Elderly in Sweden. Drugs \& Aging, 29(9), 751-758. doi:10.1007/s40266-012-0002-7

Lachamp, M., Pauly, V., Sambuc, R., Thirion, X., Potard, I., Molines, C. et Retornaz, F. (2012). Cost-effectiveness of drugs modifications for inpatients hospitalised in acute care geriatric units. La revue de médecine interne, 33(9), 482-490. doi: 10.1016/i.revmed.2012.05.013

Lau, D. T., Kasper, J. D., Potter, D. E. B., Lyles, A. et Bennett, R. G. (2005). Hospitalization and death associated with potentially inappropriate medication prescriptions among elderly nursing home residents. Archives of Internal Medicine, 165(1), 68-74. doi: 10.1001/archinte.165.1.68

Lechevalier Hurard, L. (2013). Faire face aux comportements perturbants: Le travail de contrainte en milieu hospitalier gériatrique. Sociologie du travail, 55(3), 279-301. doi 10.1016/i.soctra.2013.07.006

Luymes, C. H., van der Kleij, R. M. J. J., Poortvliet, R. K. E., de Ruijter, W., Reis, R. et Numans, M. E. (2016). Deprescribing Potentially Inappropriate Preventive Cardiovascular Medication: Barriers and Enablers for Patients and General Practitioners. The Annals of Pharmacotherapy, 50(6), 446-454. doi: 10.1177/1060028016637181

Perri, M., Menon, A. M., Deshpande, A. D., Shinde, S. B., Jiang, R., Cooper, J. W., ... Lorys, R. A. (2005). Adverse outcomes associated with inappropriate drug use in nursing homes. The Annals of Pharmacotherapy, 39(3), 405-411. doi: 10.1345/aph.1E230

Reeve, E., To, J., Hendrix, I., Shakib, S., Roberts, M. S. et Wiese, M. D. (2013). Patient barriers to and enablers of deprescribing: A systematic review. Drugs \& Aging, 30(10), 793-807. doi: 10.1007/s40266-013-0106-8

Reeve, E., Gnjidic, D., Long, J., Hilmer, S. (2015). A systematic review of the emerging definition of « deprescribing » with network analysis: implications for future research and clinical practice. British Journal of Clinical Pharmacology. décembre 2015. 80(6), 1254-1268.

Rimbert, G. (2010). Vieillards sous bonne garde : Réparer l'irréparable en maison de retraite. Broissieux, France : Éditions du Croquant. 
Van der Geest, S. et Whyte, S. R. (1989). The Charm of Medicines: Metaphors and Metonyms. Medical Anthropology Quarterly, 3(4), 345-367.

Verbeek-Heida, P. M. et Mathot, E. F. (2006). Better safe than sorry. Why patients prefer to stop using selective serotonin reuptake inhibitor (SSRI) antidepressants but are afraid to do so: Results of a qualitative study. Chronic IIIness, 2(2), 133-142. doi : 10.1177/17423953060020020801

Weber, F. (2012). Être pris en charge sans dépossession de soi ? Alter European Journal of Disability Research, 6(4), 326-339. doi: 10.1016/i.alter.2012.08.009

Emails auteurs : I.lechevalier.hurard@gmail.com ; damien.cateau@hospvd.ch ; anne.niquille@hospvd.ch ; rose-anna.foley@unisante.ch. 
Tableau 1 : Synthèse des caractéristiques des enquêtés et de leurs consommations de médicaments

\begin{tabular}{|c|c|c|c|c|c|c|c|c|c|c|c|c|}
\hline & Résidente $A$ & Résidente $B$ & Résidente C & Résidente $\mathrm{D}$ & Résidente $\mathrm{E}$ & Résidente $\mathrm{F}$ & Résidente G & Résidente $\mathrm{H}$ & Résident I & Résidente J & Résidente $\mathrm{K}$ & Résidente $\mathrm{L}$ \\
\hline Genre & Femme & Femme & Femme & Femme & Femme & Femme & Femme & Femme & Homme & Femme & Femme & Femme \\
\hline Métiers exercés & comptable & $\begin{array}{l}\text { paysanne, } \\
\text { vendeuse }\end{array}$ & $\begin{array}{l}\text { éducatrice } \\
\text { spécialisée }\end{array}$ & prof de gym & secrétaire & vendeuse & secrétaire & secrétaire & $\begin{array}{l}\text { responsable } \\
\text { technique }\end{array}$ & paysanne & $\begin{array}{c}\text { vendeuse, garde } \\
\text { barrière, } \\
\text { concierge, aide } \\
\text { agricole }\end{array}$ & $\begin{array}{c}\text { cheffe } \\
\text { d'entreprise }\end{array}$ \\
\hline Âge & 79 ans & 92 ans & 77 ans & 82 ans & 74 ans & 75 ans & 81 ans & 80 ans & 77 ans & 88 ans & 88 ans & 86 ans \\
\hline En EMS depuis & 8 ans & 3 à 4 ans & 1,5 ans & NC & 6 ans & 1,5 ans & 2 ans & 5 ans & 4 mois & 3 ans & 5 ans & 2 ans \\
\hline $\begin{array}{l}\text { Médicaments en } \\
\text { liste principale }\end{array}$ & 4 & 7 & 12 & 12 & 9 & 10 & 16 & 12 & 6 & 10 & 9 & 4 \\
\hline $\begin{array}{l}\text { Types de } \\
\text { médicaments en } \\
\text { liste principale }\end{array}$ & $\begin{array}{l}\text { neuroleptique } \\
\text { vitamines } \\
\text { traitement du } \\
\text { glaucome }\end{array}$ & $\begin{array}{l}\text { traitement de } \\
\text { l'hypertention } \\
\text { artérielle } \\
\text { traitement des } \\
\text { sécrétions acides } \\
\text { par l'estomac } \\
\text { vitamines } \\
\text { analgésique } \\
\text { traitement du } \\
\text { cholestérol } \\
\text { diurétique } \\
\text { prévention de } \\
\text { I'AVC }\end{array}$ & \begin{tabular}{|c|} 
prévention \\
cardiovasculaire \\
vitamines et \\
calcium \\
traitement de \\
l'incontinence \\
traitement du \\
diabète \\
traitement de \\
l'incontinence \\
traitement du \\
glaucome \\
traitement de \\
I'hypertension \\
artérielle \\
antiinflammatoire \\
traitement \\
hormonal \\
ménopause \\
traitement \\
ophtalmique
\end{tabular} & \begin{tabular}{|} 
hypertension \\
artérielle \\
prévention \\
cardiovasculaire \\
neuroleptique \\
vitamines et \\
calcium \\
traitement du \\
cholestérol \\
anxyolitique \\
antidépresseur \\
béta-bloquant \\
traitement du \\
diabète \\
antiinflammatoire \\
\end{tabular} & \begin{tabular}{|c} 
traitement de \\
l'anxiété \\
traitement de \\
l'incontinence \\
traitement \\
antiépileptique \\
(douleurs \\
neuropathiques) \\
bêta-bloquant \\
neuroleptique \\
antidépresseur \\
anxyolitique \\
inhibiteur de \\
sécrétions acides \\
par l'estomac \\
laxatif
\end{tabular} & \begin{tabular}{|c|} 
\\
antiépileptique \\
(douleurs \\
neuropathique) \\
antidépresseurs \\
prévention de \\
l'AVC \\
antiinflammatoire \\
diurétique \\
analgésique \\
inhibiteur des \\
secrétions acides \\
de l'estomac \\
laxatifs \\
aide à la digestion
\end{tabular} & \begin{tabular}{|c|} 
antiinflammatoire \\
prévention \\
cardiovasculaire \\
antidépresseur \\
traitement de \\
I'hypertension \\
analgésiques \\
anxyolique \\
diurétique \\
inhibiteur des \\
secrétions acides \\
de l'estomac \\
antidépresseur \\
traitement de \\
l'anxiété \\
traitement \\
antiépileptique \\
(douleurs \\
neuropathiques) \\
traitement de la \\
douleur \\
laxatif \\
\end{tabular} & $\begin{array}{l}\text { prévention } \\
\text { cardiovasculaire } \\
\text { traitement de } \\
\text { l'arthrose } \\
\text { traitement de } \\
\text { l'hypertension } \\
\text { magnesium } \\
\text { bêta-bloquant } \\
\text { inhibiteur des } \\
\text { sécretions acides } \\
\text { de l'estomac } \\
\text { antiepileptique } \\
\text { (douleurs } \\
\text { neuropathique) } \\
\text { antidépresseur } \\
\text { anxyolitique } \\
\text { traitement de la } \\
\text { mémoire } \\
\text { analgésique }\end{array}$ & $\begin{array}{c}\text { corticoïde } \\
\text { antidépresseur } \\
\text { traitements de la } \\
\text { maladie de } \\
\text { Parkinson } \\
\text { analgésique } \\
\text { diurétique } \\
\text { inhibiteur des } \\
\text { sécrétions } \\
\text { d'acides par } \\
\text { l'estomac } \\
\text { magnesium } \\
\text { traitement de } \\
\text { l'anxiété }\end{array}$ & $\begin{array}{l}\text { antidépresseur } \\
\text { prévention } \\
\text { cardiovasculaire } \\
\text { traitements de } \\
\text { l'hypertension } \\
\text { hormones } \\
\text { tyroïdiennes } \\
\text { traitement des } \\
\text { nausées } \\
\text { anxyolitique } \\
\text { analgésiques }\end{array}$ & $\begin{array}{l}\text { traitements de } \\
\text { l'ostéoporose } \\
\text { traitement de } \\
\text { I'hypertension } \\
\text { hormones } \\
\text { tyroïdiennes } \\
\text { anxyolitiques } \\
\text { traitement de la } \\
\text { maladie de } \\
\text { Parkinson } \\
\text { bêta-bloquants } \\
\text { diurétique } \\
\text { prévention de } \\
\text { l'AVC }\end{array}$ & $\begin{array}{c}\text { prévention } \\
\text { cardiovasculaire } \\
\text { laxatif } \\
\text { traitement de } \\
\text { l'agitation } \\
\text { (hypnotique) } \\
\text { diurétique }\end{array}$ \\
\hline $\begin{array}{l}\text { Traitements } \\
\text { locaux }\end{array}$ & 0 & 1 & 0 & 0 & 6 & 3 & 3 & 1 & 2 & 0 & 0 & 0 \\
\hline $\begin{array}{c}\text { Médicaments en } \\
\text { réserve }\end{array}$ & 3 & 5 & 4 & 3 & 3 & 3 & 7 & 8 & 9 & 7 & 3 & 4 \\
\hline
\end{tabular}

